\title{
ANÁLISE DO USO DE MEDICAMENTOS POTENCIALMENTE INAPROPRIADOS PARA IDOSOS EM UMA DROGARIA PRIVADA DE UM MUNICÍPIO DO VALE DO TAQUARI, RS
}

\author{
Bruna Ribeiro Xavier ${ }^{1}$, Marinês Pérsigo Morais Rigo², Luísa Scheer Ely Martines ${ }^{3}$
}

Resumo: Objetivo: Este artigo objetiva analisar os indicadores de prescrições de uma drogaria privada de um município do Vale do Taquari, RS, quanto ao uso Medicamentos Potencialmente Inapropriados (MPI) para idosos a partir da lista dos Critérios de Beers 2015. Métodos: Trata-se de um estudo transversal quantitativo por meio da análise de prescrições de pacientes idosos atendidos em uma drogaria privada do Vale do Taquari. Resultados: Foram incluídas 41 prescrições, sendo que 30 (73,17\%) são de pacientes mulheres e $11(26,83 \%)$ de homens; a média de idade dos usuários das prescrições avaliadas foi que de 68,17 anos; a média de medicamentos prescritos por prescrição foi de 2,76 medicamentos, sendo o máximo de 8 medicamentos prescritos em uma prescrição; 3 (7,32\%) prescrições haviam medicamentos com duplicidade farmacológica, sendo estes Losartana + Amilorida; 20 (48,78\%) prescrições possuíam MPI para idosos prescritos, sendo que foram encontrados 7 MPI para idosos prescritos segundo os Critérios de Beers 2015; 68,3\% das prescrições eram de usuários na faixa etária de 60 a 69 anos; 55\% das prescrições contendo MPI para idosos relacionava-se a mesma faixa etária; $26,8 \%$ das prescrições em geral e $45 \%$ das prescrições contendo MPI para idosos advinham de usuários na faixa etária entre 70 e 79 anos. Conclusão: A inclusão do profissional farmacêutico passa a ser indispensável na busca pelo uso racional dos medicamentos, responsabiliza-se pela atenção à saúde, abrangendo a totalidade da integralidade das ações de saúde.

Palavras-chave: Adesão à Medicação. Prescrições de Medicamentos. Idoso.

1 Acadêmica do Curso de Farmácia da Universidade do Vale do Taquari - Univates.

2 Farmacêutica, Mestre em Genética e Toxicologia Aplicada. Docente do Curso de Farmácia da Universidade do Vale do Taquari - Univates.

3 Farmacêutica, Doutora em Gerontologia Biomédica. Docente do Curso de Farmácia da Universidade do Vale do Taquari - Univates. 


\title{
ANALYSIS OF POTENTIALLY INAPPROPRIATE MEDICATION IN OLDER ADULTS AT A PRIVATELY HELD DRUGSTORE LOCATED IN VALE DO TAQUARI, RS
}

\begin{abstract}
Aims: This study aims to analyze the prescription indicators of a privately held drugstore located in Vale do Taquari (RS) regarding Potentially Inappropriate Medication (PMI) use in older adults according to the Beers Criteria. Methods: This is a cross-sectional, quantitative approach, through analysis, of prescriptions to older adults who buy medication at a privately held drugstore in Vale do Taquari. Results 41 prescriptions were included, of which 30 (73.17\%) belong to women and 11 (26.83\%) belong to men. Their average age was 68.17 years, and the average number of prescribed medication was of 2.76 , being 8 the maximum number for each prescription. $3(7.32 \%)$ prescriptions included medication which shares the same treatment and purpose, such as Losartan and Amiloride. 20 of them (48.78\%) included PMI for older adults, from which 7 PMI were diagnosed by the Beers Criteria. $68.3 \%$ of the prescriptions belonged to users who were from 60 to 69 years old. $55 \%$ of the prescriptions which included PMI for older adults were related to the same age group. $26.8 \%$ of the prescriptions in general and $45 \%$ of the ones containing PMI for older adults belonged to people who were from 70 to 79 years old. Conclusions: Therefore, including pharmaceutical professionals not only becomes indispensable when seeking rational, decisive use of medication, but also bears responsibility for attention to people's health, and consequently comprehending the totality and wholeness in health works.
\end{abstract}

Keywords: Medication Adherence. Drug Prescriptions. Aged.

\section{INTRODUÇÃO}

É notável o aumento da expectativa de vida na sociedade brasileira, principalmente por dois motivos: a melhoria das condições sanitárias e avanços das tecnologias médicas e farmacêuticas (AGUIAR, et al., 2008). Tais condições permitem melhor qualidade de vida e redução das taxas de mortalidade. Entretanto, a formação de políticas específicas para pessoas idosas vem se tornando altamente necessária, sendo fundamental o entendimento das necessidades e condições de vida desse segmento etário (COELHO FILHO, RAMOS, 1999; GRI, et al., 1999; LOPES, et al., 2016).

Junto com o aumento gradativo do processo de envelhecimento da população destaca-se uma maior prevalência de condições crônicas de saúde levando a uma maior probabilidade de ocorrência da polifarmácia, duplicidade farmacológica, terapia medicamentosa inadequada, reações adversas e interação entre os medicamentos (AGUIAR, et al., 2008, LUTZ, 2015).

O envelhecimento costuma ser definido por mudanças em todo processo farmacocinético que é interpretado como o que o organismo faz com o fármaco, englobando mecanismos de absorção, distribuição, metabolismo e excreção, sendo correlacionado com a perda da eficiência de muitos órgãos, assim como a diminuição da produtividade dos mecanismos homeostáticos (PARENTE, 2011). Por esta e outras particularidades do idoso, ressalta-se a necessidade de um cuidado especial junto a ele (CORSONELLO, et al., 2010) 
Diante disso, o cuidado com o Uso Racional de Medicamentos (URM) em idoso deve ser especial. Segundo Aquino (2008) a melhor maneira para se promover o URM seria primeiramente definir quando há a necessidade do uso do medicamento e em um segundo momento, o cuidado das prescrições, onde deve ser esclarecido o modo de uso, a quantidade para todo o tratamento, evitando o desperdício ou sobra do medicamento (AQUINO, 2008)

Além disso, sabe-se que a propaganda focada em promoções voltadas ao idoso, descontos e a venda livre de medicamentos possibilita o aumento do risco de intoxicações e doenças iatrogênicas resultantes da automedicação. A contribuição que o farmacêutico deve dispor ao usuário é a orientação e formação de estratégias para um aconselhamento terapêutico, ajudando a colocar em prática o URM (ARAÚJO, et al., 2008).

Por em prática o URM pode colaborar com a diminuição da polifarmácia que é definida pelo consumo de vários medicamentos e é predeterminada, pela maioria dos pesquisadores, pelo uso de cinco ou mais medicamentos por um mesmo indivíduo. A polifarmácia eleva de maneira significativa os riscos de reações adversas a medicamentos (WAWRUCH, et al., 2008; SECOLI, 2010).

A polifarmácia considerada um possível problema para o idoso, levando em conta que pacientes idosos possuem tratamentos de longa duração (LUCCHETTI, et al.; 2010). É importante salientar que os medicamentos devem ajudar na manutenção da capacidade funcional, mas também podem ser prejudiciais, pois os idosos se tornam mais suscetíveis aos efeitos adversos e às interações farmacológicas, o que intensifica quando se utiliza medicamentos inapropriados (SILVA \& ANDRADE, 2014)

Dentre os medicamentos inadequados destaca-se o termo Medicamentos Potencialmente Inapropriados (MPI) para idosos que surgiu em 1991, proposto por Beers com objetivo de determinar e prevenir a prescrição de medicamentos de alto risco para idosos e, desde então, vem sendo aprimorado e utilizado para denominar medicamentos que apresentam elevado riscos para pessoas desta classe, onde o risco sobressai o benefício (AMERICAN GERIATRICS SOCIETY, 2015). Alguns autores apontam que o uso desta classe de medicamentos tende a uma maior probabilidade de problemas relacionados aos medicamentos (PRMs) (PARENTE, 2011).

Evitar o uso de medicamentos de maior risco é uma tática importante para a diminuição de ocorrência de eventos adversos a medicamentos (FAUSTINO, et al.; 2011). A prescrição de MPI para idosos desencadeia uma maior morbilidade e mortalidade, prejudicando a qualidade de vida, ocasionando desperdícios de recursos e ainda favorecendo a polimedicação (HOLGUÍN-HERNÁNDEZ \& OROZCODÍAZ; 2010). Considera-se que cerca de um em quatro efeitos adversos são devidos ao uso de MPI (HARUGERI, et al.; 2010).

Diante disso, salienta-se a importância da interação entre o farmacêutico e paciente no momento da dispensação, pois é onde deve ocorrer a intervenção, quando necessária, 
e a orientação da terapia medicamentosa (ARAÚJO, et al., 2008). Assim, este estudo objetiva analisar prescrições contendo MPI para idosos, de acordo com os Critérios de Beers 2015 (AMERICAN GERIATRICS SOCIETY, 2015), em uma drogaria privada de um município de Vale do Taquari, RS.

\section{MATERIAIS E MÉTODOS}

Foi realizado um estudo transversal quantitativo, através da análise de prescrições de pacientes idosos atendidos em uma drogaria privada do Vale do Taquari, RS. O estudo ocorreu durante os meses de julho e agosto de 2016.

Foram incluídas no estudo prescrições advindas de pacientes com 60 anos ou mais atendidos por uma acadêmica de farmácia responsável pelo estudo. Prescrições ilegíveis foram excluídas do estudo. As prescrições incluídas no estudo foram fotocopiadas e identificadas por ordem numérica e com a idade do paciente (dado coletado conforme o manual de processos de dispensação da drogaria).

As seguintes variáveis foram coletadas:

- Número total de medicamentos: foi analisado o número total de medicamentos que constam na prescrição;

- Duplicidade farmacológica: foram avaliados quantos medicamentos apresentam a mesma composição química, mas apenas com doses diferentes;

- MPI para idosos: foram verificados quantos e quais são os medicamentos da prescrição que estão classificados como MPI para idosos conforme os Critérios de Beers 2015 (AMERICAN GERIATRICS SOCIETY, 2015);

- Idade do usuário: foi relacionado à idade do paciente com a prevalência do uso de MPI.

Os dados coletados foram tabulados em uma planilha do Microsoft Excel, onde foi realizada a frequência, média e desvio padrão das variáveis. Para relacionar a faixa etária e a quantidade de medicamentos prescritos foi utilizado o teste de Qui-Quadrado de Pearson, onde considerou-se estatisticamente significativo $\mathrm{P}<0,05$.

Foi obtida carta de autorização para realização do estudo pela gestora farmacêutica da Drogaria onde a pesquisa ocorreu. Os dados obtidos durante a pesquisa foram mantidos em sigilo pelos pesquisadores, assegurando a privacidade quanto aos dados envolvidos na pesquisa. 


\section{RESULTADOS E DISCUSSÃO}

Foram incluídas 41 prescrições, sendo que 30 (73,17\%) são prescrições de pacientes mulheres e 11 (26,83\%) de homens.

Semelhante a este trabalho a maior dimensão de prescrições do sexo feminino também se fez presente na pesquisa publicada por Neves et al., (2013) onde 75,5\% das mulheres consumiram mais medicamentos. $\mathrm{O}$ autor descreve que a mulher se preocupa mais com suas patologias e usufrui com maior frequência dos serviços de saúde, assim tornando-se mais propícias a usar o medicamento (BEERS, 1997).

Aguiar (2008), cita em seu estudo a constatação da prevalência em 21,3\% de prescrições que continham benzodiazepínicos entre mulheres com idade superior a 60 anos, mesmo sendo inadequados para as mesmas. Pesquisas recentes têm evidenciado uma série de problemas relacionados ao uso de medicamentos por idosos em diversas regiões brasileiras. Em Fortaleza, no estado do Ceará, aproximadamente 20\% dos idosos usaram ao menos um medicamento considerado inapropriado para esta idade, e em Porto Alegre, no estado do Rio Grande do Sul, em torno de 27\% dos casos foram identificados como polifarmácia (AGUIAR, et al.; 2008).

O Centro de Estudos e Avaliação em Saúde (CEFAR) da Associação Nacional das Farmácias elaborou um estudo juntamente com farmácias Portuguesas como o objetivo analisar os medicamentos utilizados por idosos. O estudo também obteve um maior número de mulheres (64,5\%), e, com uma idade média de 74,8 anos. A partir disto constatou-se que cada usuário idoso toma em média 7,3 medicamentos; $23,4 \%$ dos usuários tomam entre 4 a 5 medicamentos; $51,6 \%$ dos usuários tomam entre 6 a 9 medicamentos; $25 \%$ dos usuários tomam 10 ou mais medicamentos (PARENTE, 2011).

A média de idade dos pacientes que obtiveram as suas prescrições avaliadas foi de 68,17 anos. A média de medicamentos prescritos por prescrição foi de 2,76 medicamentos, sendo o máximo de 8 medicamentos prescritos em uma prescrição.

Cabe ressaltar que o uso de diversos fármacos não significa uma prescrição inapropriada. Porém, muitos dos indivíduos submetidos ao uso de seis ou mais fármacos, adquirem as prescrições de diferentes médicos, o que resulta em uma maior probabilidade de interação entre as prescrições e duplicidade farmacológica (LUTZ, 2015).

Diferente do encontrado neste estudo Melgaço et al., (2011), apresenta em sua pesquisa uma média de 9,2 medicamentos prescritos por idoso, porém o estudo referese à internação hospitalar. Paralelamente Carvalho et al., (2012) realizou pesquisa na região Metropolitana do Município de São Paulo com 1.114 idosos onde concluiu que $36 \%$ dos entrevistados consomem mais de 5 medicamentos.

Silveira et al., (2014) aponta que o predomínio da polifarmácia em idosos brasileiros varia de $5 \%$ a $27 \%$ e a determina como o uso conjunto de dois ou mais 
fármacos, fazendo-se uma prática contínua entre esta classe. De acordo com estudo feito em instituições asilares no nordeste do Brasil, $18,1 \%$ dos asilados utilizavam cinco ou mais medicamentos concomitantemente. Esses resultados se consolidam com dados da literatura brasileira (FLORES \& MENGUE, 2005) e de outros países uma vez que os idosos são vulneráveis às mudanças farmacocinéticas e farmacodinâmicas tornandoos predispostos a uma sucessão de reações adversas, interações medicamentosas e acompanhado da automedicação agrava ainda mais a tendência a polifarmácia (COELHO, 2016; RAJSKA-NEUMANN \& WIECZOROWSKA-TOBIS, 2007; ROLLASON \& VOGT, 2013).

O farmacêutico, ao dispensar os medicamentos, deve avaliar a prescrição e supervisionar a terapêutica medicamentosa do idoso, visando à diminuição de problemas decorrentes das interações medicamentosa (BALDONI, 2010). Segundo Secoli (2010), estas reações resultam em maiores custos assistenciais, junto aos custos de consultas a especialistas, atendimento de emergência e de internação hospitalar (SECOLI, 2010).

A excessiva ocorrência da polifarmácia na senescência torna o tratamento mais dificultado para o idoso, fazendo com que necessite de um cuidado maior para a organização de administração e horários dos medicamentos, exigindo uma memória de qualidade. Deste modo o farmacêutico torna-se um profissional de grande importância perante a sociedade, atuando junto às equipes de saúde, na melhoria da utilização dos medicamentos, no esclarecimento de dúvidas e evitando gastos desnecessários (DA SILVA \& MACEDO, 2013).

No estudo em questão, verificou-se que em $3(7,32 \%)$ prescrições haviam medicamentos com duplicidade farmacológica, sendo estas a associação de Losartana e Amilorida. Em uma avaliação de prescrições na atenção básica do Rio de Janeiro foram identificados valores mais elevados de duplicidades terapêuticas, em um total de 23,6\% dos pacientes, com média de 0,26 $\pm 0,50$ redundâncias por idoso. Entre as mulheres, este valor foi de 22,8\%, e entre os homens, $25,0 \%$. A prescrição conjunta dos medicamentos hipoglicemiantes metformina e glibenclamida condizia a 50,5\% das 101 duplicidades presentes, seguido do par losartana e enalapril (7,9\%) e ácido acetilsalicílico e clopidogrel (4,0\%) (JOÃO, 2010).

Quanto à avaliação dos MPI para idosos, 20(48,78\%) prescrições possuíam MPI prescritos, sendo que foram encontrados 7 MPI para idosos prescritos segundo os Critérios de Beers 2015 (Tabela 1). 
Tabela 1 - Relação dos medicamentos potencialmente inapropriados para idosos prescritos nas prescrições avaliadas

\begin{tabular}{l|c}
\hline \multicolumn{1}{c|}{ MPI* $^{*}$ para idoso } & Número de prescrições \\
\hline Clonazepam & 8 \\
\hline Paroxetina & 4 \\
\hline Fluoxetina & 2 \\
\hline Ácido Acetilsalicílico & 2 \\
\hline Ibuprofeno & 2 \\
\hline Espironolactona & 1 \\
\hline Digoxina Total & 1 \\
\hline \multicolumn{2}{c|}{$\mathbf{2 0}$} \\
\hline
\end{tabular}

${ }^{*}$ MPI $=$ Medicamento Potencialmente Inapropriado

Muitas pesquisas vêm ocorrendo com a intenção de notificar os MPI para utilização em idosos, tanto pela influência de efeitos adversos ou mesmo por falha da garantia e segurança do tratamento terapêutico (DE SOUZA \& ALVEZ, 2016). A tabela 2 demonstra que $68,3 \%$ das prescriçõos eram de usuários na faixa etária de 60 a 69 anos, bem como $55 \%$ das prescrições contendo MPI para idosos também relacionava-se à mesma faixa etária. Além disso, percebeu-se que $26,8 \%$ das prescrições em geral e $45 \%$ das prescrições contendo MPI para idosos advinham de usuários na faixa etária entre 70 e 79 anos. Esses dados mostraram ser estatisticamente significativos $(\mathrm{P}=0,02)$.

Tabela 2: Relação entre a faixa etária dos usuários das prescrições avaliadas e número de prescrições com medicamentos em geral e número de prescrições contendo medicamentos potencialmente inapropriados para idosos

\begin{tabular}{l|c|c}
\hline \multicolumn{1}{c|}{ Faixa etária } & $\begin{array}{c}\mathbf{N o}^{\prime} \text { prescrições } \\
\mathbf{n ~ ( \% )}\end{array}$ & $\begin{array}{c}\text { Possui prescrição com MPI } \\
\text { para idosos } \\
\mathbf{n}(\mathbf{\%})\end{array}$ \\
\hline $60-69$ anos & $28(68,3)^{*}$ & $11(55)^{*}$ \\
\hline $70-79$ anos & $11(26,8)$ & $9(45)^{*}$ \\
\hline 80 anos ou mais & $2(4,9)$ & $0(0)$ \\
\hline Total & $\mathbf{4 1 ( 1 0 0 )}$ & $\mathbf{2 0 ( 1 0 0 )}$ \\
\hline
\end{tabular}

" MPI= Medicamentos Potencialmente Inapropriados; ${ }^{*} \mathrm{P}=0,02$.

Este trabalho encontrou dados equivalentes aos resultados de uma pesquisa feita por Neves et al., 2009, ocorrida em Recife, no estado de Pernambuco. O uso de ao menos um medicamento considerado inseguro para idosos foi relatado em 21,6\% dos casos, número mais baixo do que o desta pesquisa, entretanto os principais MPI encontrados foram semelhantes: diazepam, digoxina e óleo mineral. 
Conforme estudo realizado no hospital Universitário Regional de Maringá, no estado do Paraná, foram encontrados 61 MPI para idosos entre os 253 medicamentos padronizados no hospital (aproximadamente 24\%). Semelhante ao estudo abordado neste trabalho foi verificado que uma grande maioria desses MPI, como benzodiazepínicos, antidepressivos e relaxantes musculares, estão comprometidos em muitas reações adversas, o que comprova que devem ser utilizados com cautela em faixas etárias mais avançadas (GRISHAW, et al., 2001, STROHER \& ZUBIOLI, 2014).

A grande maioria dos medicamentos usados pelos idosos são, na maior parte, obtidos na rede pública de saúde, alguns similares sugeridos não estão disponíveis para uso, já que abrangem alternativas terapêuticas não existentes na Relação Nacional de Medicamentos Especiais (RENAME) nem na Relação Municipal de Medicamentos Essenciais (REMUME), tais como o lorazepam e a sertralina. Isto pode cooperar para a falta de sucesso da intervenção farmacêutica. Dados como este indicam a urgência da implantação de equivalentes terapêuticos nas listas municipais e nacionais, que sejam adequados para os idosos oferecendo maior segurança (STROHER \& ZUBIOLI, 2014).

De acordo com o estudo realizado no Hospital Beneficência Portuguesa de São Paulo, estado de São Paulo, também houve um número grande de MPI para idosos, com prevalência de $35,47 \%$ na amostragem total, e os principais MPI encontrados foram: ácido acetilsalicílico (22,27\%), espironolactona (12,63\%), cloridrato de amiodarona (4,09\%), glibenclamida (2,27\%), lorazepam/clonazepam $(2,27 \%)$ e metildopa $(1,36 \%)$ (GUIMARÃES \& MOURA, 2012).

\section{CONCLUSÃO}

O uso de medicamentos aumenta com o envelhecer e, dessa forma, o excesso de fármacos utilizados aumenta a probabilidade do uso de algum MPI para idosos. Visto que quase $50 \%$ das prescrições analisadas continha um MPI para idoso prescrito, percebe-se a importância de buscar critérios clínicos para auxiliar na seleção da terapêutica entre as opções disponíveis.

Devem ser evitados todos aqueles medicamentos em que o risco excede o benefício, sendo de suma importância dispor de conhecimento sobre os MPI para idosos e prever intervenções que busquem uma melhor estratégia neste grupo populacional.

Considerando que o farmacêutico é o último a ter contato com o paciente antes da utilização da terapia medicamentosa e que este pode interceder em prescrições inadequadas e problemas encontrados no receituário, o conhecimento dos Critérios de Beers torna-se útil para a identificação de erros, obrigando-se a alertar o paciente e o médico responsável. Assim a inclusão do profissional farmacêutico passa a ser indispensável na busca pelo uso racional e determinante dos medicamentos, 
responsabilizando-se também pela atenção à saúde, e buscando abranger a totalidade da integralidade das ações de saúde.

\section{REFERÊECIA}

AGUIAR P.M.; LYRA JUNIOR, D.P.;, SILVA, D.T.; et al. Avaliação da farmacoterapia de idosos residentes em instituições asilares no nordeste do Brasil. Latin American Journal of Pharmacy, v. 27, n. 3, p. 254-259, 2008.

AMERICAN GERIATRICS SOCIETY. Critérios de Beers 2015: versão atualizada e expandida. São Paulo: Sociedade Brasileira de Geriatria e Gerontologia, 2015.

AQUINO, D, S. Porque o uso racional de medicamentos deve ser uma prioridade? Revista Ciência \& Saúde Coletiva, Recife, v. 13, p. 733-736, abr. 2008.

ARAUJO, A. L. A. et al. Perfil da assistência farmacêutica na atenção primária do Sistema Único de Saúde. Ciência \& Saúde Coletiva, v. 13, p. 611-617, 2008.

BALDONI, A. O. Estudo de utilização de medicamentos em idosos atendidos pelo sistema único de saúde SUS. 2010. 133f. Dissertação (Mestrado em Ciências) - Faculdade de Ciências Farmacêuticas de Ribeirão Preto - Universidade de São Paulo. Ribeirão Preto, 2010.

BEERS, M. H. Explicit criteria for determining potentially inappropriate medication use by the elderly. Archives of Internal Medicine., v. 157, n. 14, p.1531-1536, 1997.

CARVALHO, M.F.C.; ROMANO-LIEBER, N.S.; BERGSTEN-MENDES, G.; et al. Polifarmácia entre idosos do Município de São Paulo-Estudo SABE. Revista Brasileira de Epidemiologia, vol. 15, n. 4, p. 817-827, 2012.

COELHO FILHO, J. M.; RAMOS, L. R. Epidemiologia do envelhecimento no Nordeste do Brasil: resultados de inquérito domiciliar. Revista de Saúde Pública, São Paulo, v. 33, n. 5, p. 445-453, 1999.

COELHO, A.R.C. Farmacocinética da Geriatria [dissertação]. Algada, Portugal: Instituto Superior de Ciências da Saúde Egas Moniz; 2016.

CORSONELLO, A.; PEDONE, C.; INCALZI, R.A. Age-related pharmacokinetic and pharmacodynamic changes and related risk of adverse drug reactions. Current Medicinal Chemistry, v. 17, n. 6, p. 571-84, 2010.

DA SILVA, E.L.; MACEDO, L.C. Polifarmácia em Idosos. Saúde e Pesquisa, n.6, v.3, p.477-486, 2013.

DE SOUZA, P.; ALVES, S. Investigação farmacoepidemiológica em prescrições médicas da Atenção Básica: o caso do idoso. Academus Revista Científica da Saúde, n.1, v.1, p. 3-14, 2016.

FAUSTINO, C. G.; MARTINS, M. A.; JACOB-FILHO, W. Medicamentos potencialmente inapropriados prescritos a pacientes idosos ambulatoriais de clínica médica. Einstein (São Paulo), v. 9, n. 1, p. 18-23, 2011. 
FLORES, L.M,; MENGUE, S.S. Uso de medicamentos por idosos em região do sul do Brasil. Revista de Saúde Pública, vol.39, n.6, p. 924-929, 2005.

GRI, E. et al. A. Consumo de medicamentos y remedios naturales en la población anciana de un área rural. Atención Primaria, Barcelona, v.23, n.8, p.455-460, 1999.

GRISHAW, J.M.; SHIRRAN, L.; THOMAS, R.; et al. Changing provider behavior: an overview of systematic reviews of interventions. Medical Care, vol. 39, n. 9, Supl 2, p.II2-45, 2001.

GUIMARÃES, P.L.; MOURA, C.S. Fatores Associados ao Uso de Medicamentos Impróprios de Alto Risco em Pacientes Idosos Hospitalizados. Revista Brasileira de Farmácia Hospitalar e Serviços de Saúde, v. 3, n.4, p. 9-15, 2012.

HARUGERI, A. et al. Potentially inappropriate medication use in elderly patients: a study of prevalence and predictors in two teaching hospitals. Journal of Postgraduate Medicine. v. 56, n. 3, p. 186-191, 2010.

HOLGUÍN-HERNÁNDEZ, E.; OROZCO-DÍAZ, J.G. Medicación potencialmente inapropriada en ancianos en un hospital de primer nível, Bogotá 2007. Revista de Salud Pública. v. 12, n. 2, p. 287-299, 2010.

JOÃO, W. S. J. Reflexão sobre o uso racional de medicamentos. Pharmacia Brasileira, n. 78, set./out, 2010.

LOPES, L.M.; FIGUEIREDO, T.P.; COSTA, S.C.; et al. Use of potentially inappropriate medications by the elderly at home. Ciência \& Saúde Coletiva, v. 21, n. 11, p. 3429-3438, 2016.

LUCCHETTI, G.; et al. Fatores associados à polifarmácia em idosos institucionalizados Revista Brasileira de Geriatria e Gerontologia, v. 13, n. 1, p. 51-58, jan./abr. 2010.

LUTZ, B. H. Inadequação do uso de medicamentos entre idosos na cidade de Pelotas, RS. 2015. 144f. Dissertação (Mestrado) - Universidade Federal de Pelotas. Pelotas, 2015.

MELGAÇO, T.B.; CARRERA, J.K.; NASCIMENTO, D.E.B.; et al. Polifarmácia e ocorrências de possíveis interações medicamentosas. Revista Paraense de Medicina, v.25, n.1, p. 1-8, 2011.

NEVES, S.J.F.; MARQUES, A.P.O.; LEAL, M.C.C.; et al. Epidemiologia do uso de medicamentos entre idosos em área urbana do Nordeste do Brasil. Revista de Saúde Pública, v.47, n.4, p. 759-768, 2013.

PARENTE, J. P. O. Avaliação do uso de medicamentos inapropriados em idosos: aplicação dos critérios de Beers. 2011. 75f. Dissertação (Mestrado). Ciências Farmacêuticas. Faculdade de Ciências da Saúde. Universidade da Beira Interior. Covillã. 2011.

RAJSKA-NEUMANN, A.; WIECZOROWSKA-TOBIS, K. Polypharmacy and potential inappropriateness of pharmaco-logical treatment among community-dwelling elderly patients. Archives of Gerontology and Geriatrics, vol. 44, p. 303-309, 2007. 
ROLLASON, V; VOGT, N. Reduction of polypharmacy in the elderly: a systematic review of the role of the pharmacist. Drugs Aging, v.20, n.11, p. 817-832, 2013.

SECOLI, S. R. Polifarmácia: Interações e reações adversas no uso de medicamentos por idosos. Revista Brasileira de Enfermagem, v. 63, n.1, p. 136140, Jan./Feb., 2010.

SILVA, D. P. T.; ANDRADE, J. A. Atenção farmacêutica ao idoso no uso da polifarmácia. 2014. 29f. Monografia (Graduação). Curso de Farmácia. Universidade de Pindamonhangaba. São Paulo. 2014.

SILVEIRA, E.A.; DALASTRA, L.; PAGOTTO, V.; et al. Polifarmácia, doenças crônicas e marcadores nutricionais em idosos. Revista Brasileira de Epidemiologia, v. 17, n.4, p. 818829, 2014.

STROHER, A.; ZUBIOLI, A. Prevalência de medicamentos potencialmente inapropriados para idosos entre os padronizados no Hospital Universitário Regional de Maringá de acordo com os critérios de Beers-Fick. Infarma-Ciências Farmacêuticas, v. 26, n.1, p.4-10, 2014.

WAWRUCH, M.; FIALOVA, D.; ZIKAVSKA, M. et al. Factors influencing the use of potentially inappropriate medication in older patients in Slovakia. Journal of clinical pharmacy and therapeutics, vol. 33, n.4, p.381-392, 2008. 